\title{
Lobar pneumonia caused by Ralstonia pickettii in a sixty-five-year-old Han Chinese man: a case report
}

\author{
Wensen Pan ${ }^{1}$, Zhiming Zhao ${ }^{2^{*}}$ and Mei Dong ${ }^{3}$
}

\begin{abstract}
Introduction: Ralstonia pickettii is a gram-negative, oxidase-positive bacillus and is an emerging pathogen found in infections described in hospital settings. The cases reported in the literature mostly are nosocomial infections due to contaminated blood products, sterile water, saline, treatment fluids and venous catheters. Human infection unrelated to contaminated solutions is rare. We report a case of lobar pneumonia and pulmonary abscess caused by Ralstonia pickettii in an older patient.

Case presentation: A sixty-five-year old Han Chinese man presented having had cough, expectoration, chest pain and fever lasting for twenty days. His medical history was notable for hypertension over the previous ten years, and the habit of smoking for forty years. A thoracic computed tomography scan supported the diagnosis of rightsided lobar pneumonia. A lung biopsy was done and pathological analysis confirmed lobar pneumonia. Two lung biopsy specimens from separate sites grew Ralstonia pickettii. After six days, a repeat thoracic scan revealed a rightsided abscess. A thoracentesis was performed and the purulent fluid grew Ralstonia pickettii. The chest tube remained inserted to rinse the cavity with sterile sodium chloride. He received an antibiotic course of intravenous cefoperazone sodium-sulbactam sodium for eighteen days and imipenem-cilastatin for twelve days. A repeat chest $X$-ray revealed resolution of the pulmonary abscess and improvement of pneumonia. He remained afebrile and free of respiratory symptoms after treatments.
\end{abstract}

Conclusion: This case demonstrates a Ralstonia pickettii infection in the absence of an obvious nosocomial source. It is possible that such cases will become common in the future. Therefore, further studies are needed to evaluate its sensitivity to common antibiotics.

\section{Introduction}

Ralstonia pickettii ( $R$. pickettii) is an emerging pathogen. It is ubiquitous in nature and is found naturally in soil and groundwater. R. pickettii was first isolated in 1973 and included in the genus Pseudomonas [1]. The rodshaped organism was reclassified in the Burkholderia and then the Ralstonia genera, receiving its current name in 1995 [2]. R. pickettii is often confused with other similar bacteria, increasing the difficulty of classifying and treating this pathogen.

$R$. pickettii can be isolated from various clinical specimens, such as sputum, blood, infected wounds, urine,

\footnotetext{
* Correspondence: pwszzmxx@sina.com.cn

${ }^{2}$ Department of Reproductive Medicine, the Second Hospital of Hebei

Medical University, Shijiazhuang, China

Full list of author information is available at the end of the article
}

ear, nose swabs, and cerebrospinal fluid. It is also commonly found in the respiratory tract secretions of cystic fibrosis patients. Most infections with $R$. pickettii are seen in the hospital setting resulting in bacteremia and/ or septicemia and respiratory infections and/or pneumonia [3]. The cases reported in the literature are mostly nosocomial infections due to contaminated solutions including blood products, sterile water, saline, chlorhexidine solution, treatment fluids for the respiratory tract, and contaminated venous catheters [4-7]. Human infection unrelated to contaminated solutions is rare. There is only one documented case of an empyema caused by R. pickettii, and our case is similar in some respects [8]. Our case is perhaps the first one reported of a lobar pneumonia and pulmonary abscess caused by $R$. pickettii.

\section{Biomed Central}

(C) 2011 Pan et al; licensee BioMed Central Ltd. This is an Open Access article distributed under the terms of the Creative Commons Attribution License (http://creativecommons.org/licenses/by/2.0), which permits unrestricted use, distribution, and reproduction in any medium, provided the original work is properly cited. 


\section{Case presentation}

A sixty-five-year old Han Chinese man presented with cough, expectoration, chest pain and fever lasting twenty days to the emergency room. His medical history was notable for hypertension over the previous ten years, and a forty-year smoking history (20 cigarettes per day). He did not have previous exposure to respiratory therapy solutions and had not taken any antimicrobial agents in the past five years. Pleuritic chest pain was the most prominent symptom. The cough was dry initially, but about five days later became productive. He had a sudden onset of a high fever to $39^{\circ} \mathrm{C}$, which resulted in rigors. Upon initial presentation, he received antibiotic therapy of intravenous penicillin sodium for seven days, lavo-ofloxacin for five days and cefotaxime sodium for seven days in turn, but his symptoms did not improve, resulting in admission to our hospital.

His initial physical examination upon admission revealed a temperature of $38.8^{\circ} \mathrm{C}$; blood pressure, $120 / 85$ $\mathrm{mmHg}$; respiratory rate, 23 breaths per minute; and pulse, 90 beats per minute. Evaluation for a source of the fever demonstrated a right-sided lobar pneumonia on chest X-ray. A thoracic computed tomography (CT) scan supported the diagnosis of right-sided lobar pneumonia (Figure 1). Subsequently, a CT-guided lung biopsy was done, which confirmed it to be lobar pneumonia in the period of gray hepatization (Figure 2). Two biopsy specimens from separate sites grew $R$. pickettii in pure cultures, which was identified by the API $20 \mathrm{NE}$ system (bioMérieux France, No.0041445). He received an antibiotic course of intravenous cefepime for six days. A repeat thoracic CT scan revealed the presence of a right-sided abscess (Figure 3). A centesis was

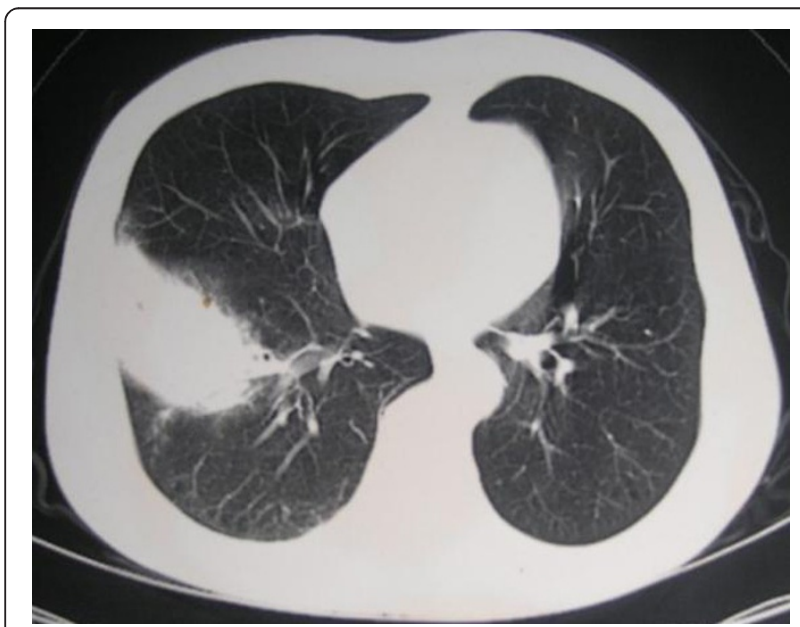

Figure 1 Computed tomography scan (GE Medical System. lightspeed 16) of the thorax showing features of lobar pneumonia. In this image right lung lower lobe soft tissue density shadow can be seen.

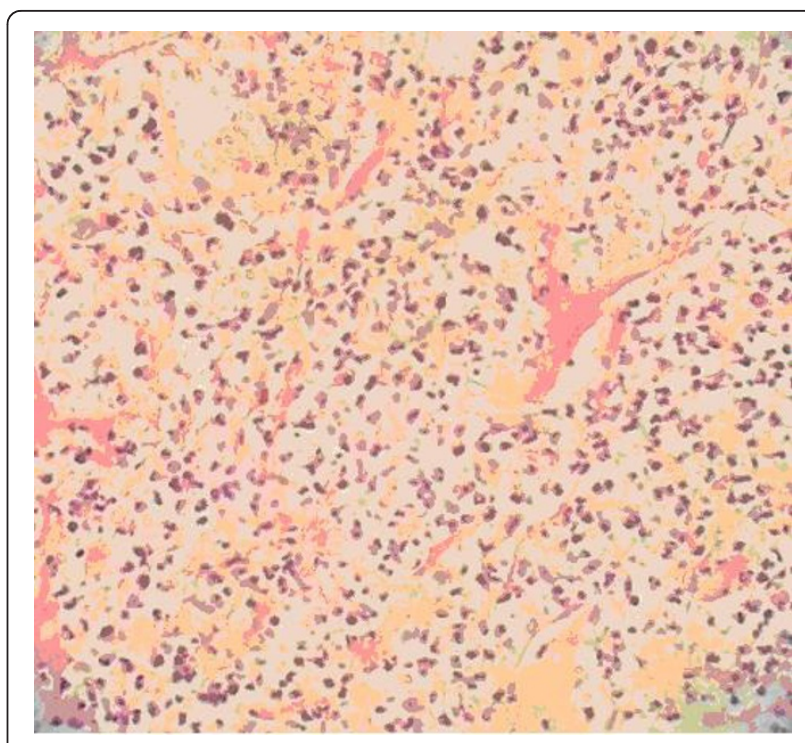

Figure 2 Lung biopsy appearance of lobar pneumonia-like changes of the gray phase of liver. It is showing that alveolar space is clearly visible, a large number of cellulose can be seen seeping into cavity to form a network and through Trichoderma Kong mutual links with the neighboring alveolar space. (hematoxylin and eosin, magnification $\times 40$ ).

performed and the purulent fluid grew $R$. pickettii. The chest tube placed for drainage remained in the cavity of the abscess for rinsing with sterile sodium chloride solution. Because the pathogen had not responded to two antibiotic treatments, its antimicrobial susceptibilities were studied by the disk diffusion method of the Clinical and Laboratory Standards Institute (CLSI) (9). The breakpoints used to determine resistance and

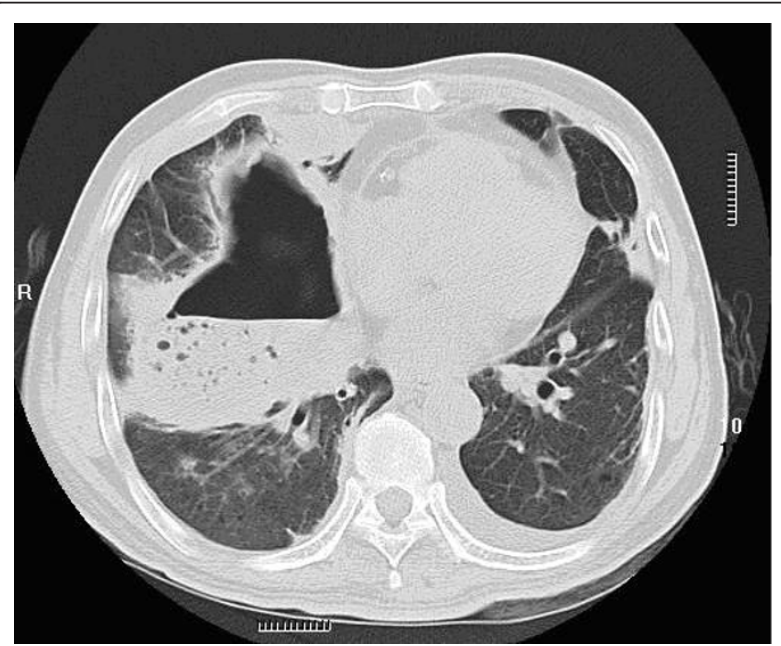

Figure $\mathbf{3}$ Computed tomography scan of the thorax. The scan shows features typical of pulmonary abscesses, consolidation with a single cavity containing an air-fluid level in the right lung after six days of intravenous cefepime treatment. 
susceptibility to the antibiotics were provided simultaneously (Table 1 ). In this case, the $R$. pickettii isolate was susceptible to cefoperazone sodium-sulbactam sodium, ceftazidime, and imipenem according to the disk diffusion method. The pathogen was resistant to amikacin, ceftriaxone, ciprofloxacin, mezlocillin, aztreonam, and gentamicin. Our patient received an antibiotic course of intravenous cefoperazone sodium-sulbactam sodium for eighteen days and imipenem-cilastatin for twelve days. A repeat chest X-ray performed forty-eight days later revealed resolution of the pulmonary abscess and improvement of pneumonia. The patient remained afebrile and free of respiratory symptoms at follow-up two months later.

\section{Discussion}

Human infection with $R$. pickettii without exposure to contaminated solutions is rare and isolation of the organism in culture alone is often attributed to laboratory contamination rather than to infection. Therefore, infection with $R$. pickettii is typically diagnosed when treatment targeting the organism and/or removal of an infected source is associated with clinical improvement. For example, a recently reported case of $R$. pickettii infection in a pediatric oncology unit described clinical improvement only with catheter removal and appropriate antimicrobial therapy [10]. In this case, isolation of $R$. pickettii in culture from a sterile site coupled with clinical improvement following thoracentesis and targeted antimicrobial therapy increases the likelihood that the organism was the pathogenic source. In our case, there had been no use of respiratory therapy solutions excluding the possibility of exposure to fluids contaminated with $R$. pickettii.

$R$. pickettii is generally believed not to be the primary pathogen and, alone, its infectivity is very low. Recent reports show that it can lead to a number of potentially serious infections, nosocomial outbreaks $[4,11]$ and even death [3]. Antimicrobial susceptibility patterns reported for $R$. pickettii vary widely. $R$. pickettii can produce extended-spectrum $\beta$-lactamases, which are not commonly sensitive to inhibitors of $\beta$ lactamase [12-15]. They show that the organism is resistant in different degrees to ciprofloxacin, trimethoprim-pyrimidine, sulfamethoxazole, piperacillintazobactam, imipenem and cilastatin, ceftazidime. Following susceptibility studies, our patient was successfully treated with intravenous cefoperazone sodiumsulbactam sodium for eighteen days and imipenemcilastatin for twelve days.

\section{Conclusion}

We describe the case of an older man who developed $R$. pickettii infection in the absence of an obvious nosocomial source demonstrating the possibility that such de novo cases will become more common in the future. Although it is of low virulence, it has been identified as causing many potentially harmful infections, and even death. The pathogen was resistant to many antibiotics, so its sensitivity to the common antibiotics should be monitored regularly.

\section{Consent}

Written informed consent was obtained from the patient for publication of this case report and accompanying images. A copy of the written consent is available for review by the Editor-in-Chief of this journal.

\section{Abbreviations}

CLSI: Clinical and Laboratory Standards Institute; CT: computed tomography; R. Pickettii: Ralstonia pickettii.

\section{Acknowledgements}

We thank Baofa Wang, Yadong Yuan (Hebei Medical University), Jessica A. Hennessey and Chuan Wang (Duke University Medical Center) for helping in writing this manuscript.

\section{Author details}

Department of Respiratory Medicine, the Second Hospital of Hebei Medical University, Shijiazhuang, China. ${ }^{2}$ Department of Reproductive Medicine, the Second Hospital of Hebei Medical University, Shijiazhuang, China.

${ }^{3}$ Department of Internal Medicine, the Affiliated Hospital of Hebei University of Science and Technology, Shijiazhuang, China.

Table 1 Breakpoints used to determine resistance and susceptibility to antimicrobial therapy

\begin{tabular}{|c|c|c|c|c|}
\hline Antimicrobial Agent & Disk Content & & kpoints, & \\
\hline & & $S$ & 1 & $\mathbf{R}$ \\
\hline mezlocillin & $75 \mu \mathrm{g}$ & $\geqq 16$ & - & $\leqq 15$ \\
\hline ceftazidime & $30 \mu \mathrm{g}$ & $\geqq 18$ & $15-17$ & $\leqq 14$ \\
\hline cefoperazone & $75 \mu \mathrm{g}$ & $\geqq 21$ & $16-20$ & $\leqq 15$ \\
\hline ceftriaxone & $30 \mu \mathrm{g}$ & $\geqq 21$ & $14-20$ & $\leqq 13$ \\
\hline imipenem & $10 \mu \mathrm{g}$ & $\geqq 16$ & $14-15$ & $\leqq 13$ \\
\hline aztreonam & $30 \mu \mathrm{g}$ & $\geqq 22$ & $16-21$ & $\leqq 15$ \\
\hline gentamicin & $10 \mu \mathrm{g}$ & $\geqq 15$ & $13-14$ & $\leqq 12$ \\
\hline ciprofloxacin & $5 \mu \mathrm{g}$ & $\geqq 21$ & $16-20$ & $\leqq 15$ \\
\hline
\end{tabular}




\section{Authors' contributions}

WP collected the patient data and was a major contributor in writing the manuscript. ZZ and MD performed CT-guided lung biopsy and the histological examination of the lung. All authors read and approved the final manuscript.

\section{Competing interests}

The authors declare that they have no competing interests.

Received: 14 April 2010 Accepted: 15 August 2011

Published: 15 August 2011

\section{References}

1. Ralston E, Palleroni NJ, Doudoroff M: Pseudomonas pickettii, a new species of clinical origin related to Pseudomonas solanacearum. Int J Syst Bacteriol 1973, 23:15-19.

2. Yabuuchi E, Kosako Y, Yano I, Hotta H, Nishiuchi Y: Transfer of two Burkholderia and an Alcaligenes species to Ralstonia gen. nov.: proposal of Ralstonia pickettii (Ralston, Palleroni and Doudoroff 1973) comb. nov., Ralstonia solanacearum (Smith 1896) comb.nov. and Ralstonia eutropha (Davis 1969) comb.nov. Microbiol Immunol 1995, 39:897-904.

3. Ryan MP, Pembroke JT, Adley CC: Ralstonia pickettii: a persistent Gramnegative nosocomial infectious organism. J Hosp Infect 2006, 62:278-284.

4. Moreira BM, Leobons MB, Pellegrino FL, Santos M, Teixeira LM, de Andrade Marques E, Sampaio JL, Pessoa-Silva CL: Ralstonia pickettii and Burkholderia cepacia complex bloodstream infections related to infusion of contaminated water for injection. J Hosp Infect 2005, 60:51-55.

5. Labarca JA, Sader HS, Peterson CL, Carson LA, Holt SC, Arduino MJ, Meylan M, Mascola L, Jarvis WR: A multistate nosocomial outbreak of Ralstonia pickettii colonization associated with an intrinsically contaminated respiratory care solution. Clin Infect Dis 1999, 29:1281-1286.

6. McNeil MM, Solomon SL, Anderson RL, Davis BJ, Spengler RF, Reisberg BE, Thornsberry C, Martone WJ: Nosocomial Pseudomonas pickettii colonization associated with a contaminated respiratory therapy solution in a special care nursey. J Clin Microbiol 1985, 22:903-907.

7. Yoneyama A, Yano H, Hitomi S, Okuzumi K, Suzuki R, Kimura S: Ralstonia pickettii colonization of patients in an obstetric ward caused by a contaminated irrigation system. J Hosp Infect 2000, 46:79-80.

8. Wills TS, Lopez J, Billington AR: Empyema Caused by Ralstonia pickettii in a Hemodialysis Patient. Clin Microbiol Newsletter 2007, 29:55-56.

9. Clinical and Laboratory Standards Institute: Performance Standards for Antimicrobial Susceptibility Testing; Eighteenth Informational Supplement Wayne, PA; 2007, (ISBN 1-56238-625-5).

10. Kismet E, Atay AA, Demirkaya E, Aydin HI, Aydogan H, Koseoglu V, Gokcay E: Two cases of Ralstonia pickettii bacteremias in a pediatric oncology unit requiring removal of the Port-A-Caths. J Pediatr Hematol Oncol 2005, 27:37-38.

11. Kimura AC, Calvet H, Higa Jl, Pitt H, Frank C, Padilla G, Arduino M, Vugia DJ: Outbreak of Ralstonia pickettii Bacteremia in a Neonatal Intensive Care Unit. Pediatr Infect Dis J 2005, 24:1099-1103.

12. Girlich D, Naas T, Nordmann P: OXA-60, a chromosomal, inducible, and imipenem-hydrolyzing class $D \beta$-lactamase from Ralstonia pickettii. Antimicrob Agents Chemother 2004, 48:4217-4225.

13. Nordmann P, Poirel L, Kubina M, Casetta A, Naas T: Biochemical-genetic characterization and distribution of OXA-22, a chromosomal and inducible class D $\beta$-lactamase from Ralstonia (Pseudomonas) pickettii. Antimicrob Agents Chemother 2000, 44:2201-2204.

14. Sader HS, Jones RN: Antimicrobial susceptibility of uncommonly isolated non-enteric gram-negative bacilli. Int J Antimicrob Agents 2005, 25:95-109.

15. Gales AC, Jones RN, Andrade SS, Sader HS: Antimicrobial susceptibility patterns of unusual nonfermentative gram-negative bacilli isolated from Latin American: report from the SENTRY Antimicrobial Surveillance Program (1997-2002). Mem Inst Oswaldo Cruz, Rio de Janeiro 2005, 100:671-677.

doi:10.1186/1752-1947-5-377

Cite this article as: Pan et al.: Lobar pneumonia caused by Ralstonia pickettii in a sixty-five-year-old Han Chinese man: a case report. Journal of Medical Case Reports 2011 5:377.

\section{Submit your next manuscript to BioMed Central and take full advantage of:}

- Convenient online submission

- Thorough peer review

- No space constraints or color figure charges

- Immediate publication on acceptance

- Inclusion in PubMed, CAS, Scopus and Google Scholar

- Research which is freely available for redistribution 